\title{
Influencia del mercado canadiense en el turismo cubano
}

DOI: https://doi.org/10.33262/ap.v1i2.8

\section{Influence of the Canadian market on Cuban tourism}

Dariel Armstrong Zulueta. ${ }^{1}$ \& Melissa Lemes Reyes. ${ }^{2}$

\begin{abstract}
.
The tourism sector in Cuba has been the main generator of the economy in the new century. Tourism income has been marked by the constant growth of tourist arrivals that Cuba has, reaching figures of almost 5 million people. Canadian tourism that travels to the island plays a fundamental role in these indices. Canada has historically stood out for being the main source market for tourists to Cuba, with current figures of approximately $30 \%$ of the total number of tourists issued by the main markets, which represent more than $60 \%$ of the total number of tourists arriving in Cuba. For this reason, special attention has been paid to the study of this type of client to maintain their preference among Caribbean destinations. Knowing the significance of this market is vital for programming and estimating the tourism sector in general, since a fall in Canadian arrivals would affect the tourism economy more than that of any other source market. The following research aims to apply econometric models to Canadian demand to estimate and forecast the incidence of fluctuations in this market on international tourist arrivals in the country. For this, mathematical and statistical methods are used from computer tools such as Microsoft Excel and SPSS. With them, correlational analyzes, multiple regression, and linear programming were carried out that allowed us to know and estimate the behavior of international tourism on the island from the influence of Canadian tourism.
\end{abstract}

\footnotetext{
${ }^{1}$ Universidad de La Habana. Facultad de Turismo. La Habana, Cuba. smfdariel@gmail.com

${ }^{2}$ Universidad de La Habana. Facultad de Turismo. La Habana, Cuba. darielarmstrong@gmail.com
} 
Keywords: Canada, programming, estimation, SPSS, Mathematical and statistical models.

\section{Resumen.}

El sector turístico en Cuba ha sido el principal motor generador de la economía en el nuevo siglo. Los ingresos turísticos han sido marcados por el constante crecimiento de los arribos turísticos que tiene Cuba, alcanzando cifras de casi 5 millones de personas. En estos índices juega un papel fundamental el turismo canadiense que viaja a la isla. Canadá se ha destacado históricamente por ser el principal mercado emisor de turistas a Cuba, con cifras actuales de aproximadamente el 30\% del total de turistas emitidos por los principales mercados, los cuales representan más del 60\% del total de turistas llegados a Cuba. Por esta razón se le ha prestado especial atención al estudio de este tipo de clientes para mantener su preferencia entre los destinos del Caribe. Conocer la significación que tiene este mercado es vital para la programación y estimación del sector turístico en general, puesto que una caída de los arribos canadienses afectaría la economía turística más que la de cualquier otro mercado emisor. La siguiente investigación tiene como objetivo la aplicación de modelos econométricos a la demanda canadiense para estimar y pronosticar la incidencia de las fluctuaciones de este mercado en los arribos turísticos internacionales del país. Para ello se emplean métodos matemáticos y estadísticos a partir de herramientas informáticas como el Microsoft Excel y el SPSS. Con ellos se realizaron análisis correlaciónales, de regresión múltiple, y programación lineal que permitieron conocer y estimar el comportamiento del turismo internacional en la isla a partir de la influencia del turismo canadiense.

Palabras claves: Canadá, programación, estimación, SPSS, Modelos matemáticos y estadísticos.

\section{Introducción.}

La creación de flujos turísticos crecientes y de magnitud significativa es el principal objetivo del desarrollo de los destinos turísticos (Martín Fernández, 2006). En este sentido Canadá ha jugado un papel fundamental en el desarrollo del destino Cuba. Las relaciones entre Cuba y Canadá se han unificado desde muchas aristas distintas. El turismo ha sido una que ha fortalecido los lazos que unen a estos dos pueblos. El mercado canadiense ha sido para Cuba de vital importancia desde la apertura al turismo como sector impulsor de la economía del país y es por esta razón que es seguido el comportamiento de este mercado para mantener sus índices de satisfacción.

Es del conocimiento de las direcciones del turismo del país caribeño la importancia de este mercado, pero: ¿es realmente conocida la incidencia que tiene este mercado en las fluctuaciones turísticas que sufre el país? Constantemente se están haciendo estimaciones turísticas para trazar objetivos estratégicos de cumplimiento con vistas anuales; pero ¿se 
tienen en cuenta realmente las variables de las que dependen los arribos turísticos generales para esas estimaciones?

A partir de las interrogantes anteriores se formula el siguiente problema de investigación:

¿Qué incidencia tiene el mercado canadiense como variable independiente dentro de los arribos turísticos de los principales mercados que se realizan anualmente en Cuba, y de qué forma se podrían hacer pronósticos más certeros teniendo en cuenta esta variable?

Para poder responder la anterior interrogante problemática se trazó el siguiente:

\section{Objetivo general:}

- Evaluar la incidencia que tiene los arribos del mercado canadiense en la estimación de arribos turísticos mensuales de los principales mercados emisores de turismo a Cuba.

\section{Objetivos específicos:}

- Determinar los métodos y herramientas estadísticas necesarias para el análisis.

- Diagnosticar matemáticamente la incidencia del mercado canadiense en los arribos turísticos.

\section{Metodología:}

Para el cumplimiento de los objetivos trazados se emplearon como herramientas fundamentales el software informático Microsoft Excel, y el estadístico SPSS.

Microsoft Excel: Desde la obtención de la información suministrada por el MINTUR se utilizó esta herramienta ya que los datos recopilados se encontraban en tablas de este programa. Con esta herramienta se filtró la información necesaria para la investigación, se organizó para un mejor aprovechamiento de la misma, se exportaron los datos para el programa SPSS para su posterior análisis estadístico, se importaron los resultados para una mejor organización de estos, y se exportaron nuevamente para la conformación del informe de la investigación.

SPSS: Con este programa se aplicaron modelos econométricos para determinar todas las bases que sustentan esta investigación. Arrojó los resultados necesarios a partir de la correlación de variables, la regresión múltiple y la programación lineal, como principales herramientas estadísticas con las cuales se alcanzaron los objetivos propuestos.

\section{Resultados:}

Los datos trabajados fueron suministrados por el MINTUR; estos son referidos a la llegada de turistas de los principales mercados a Cuba por meses en los años 2017, 2018, y 2019. De 
ISSN: 2773-7330

estos se sacaron las llegadas canadienses a Cuba, y el total de turistas en los meses de estos años.

Tabla 1. Llegada de turistas a Cuba.

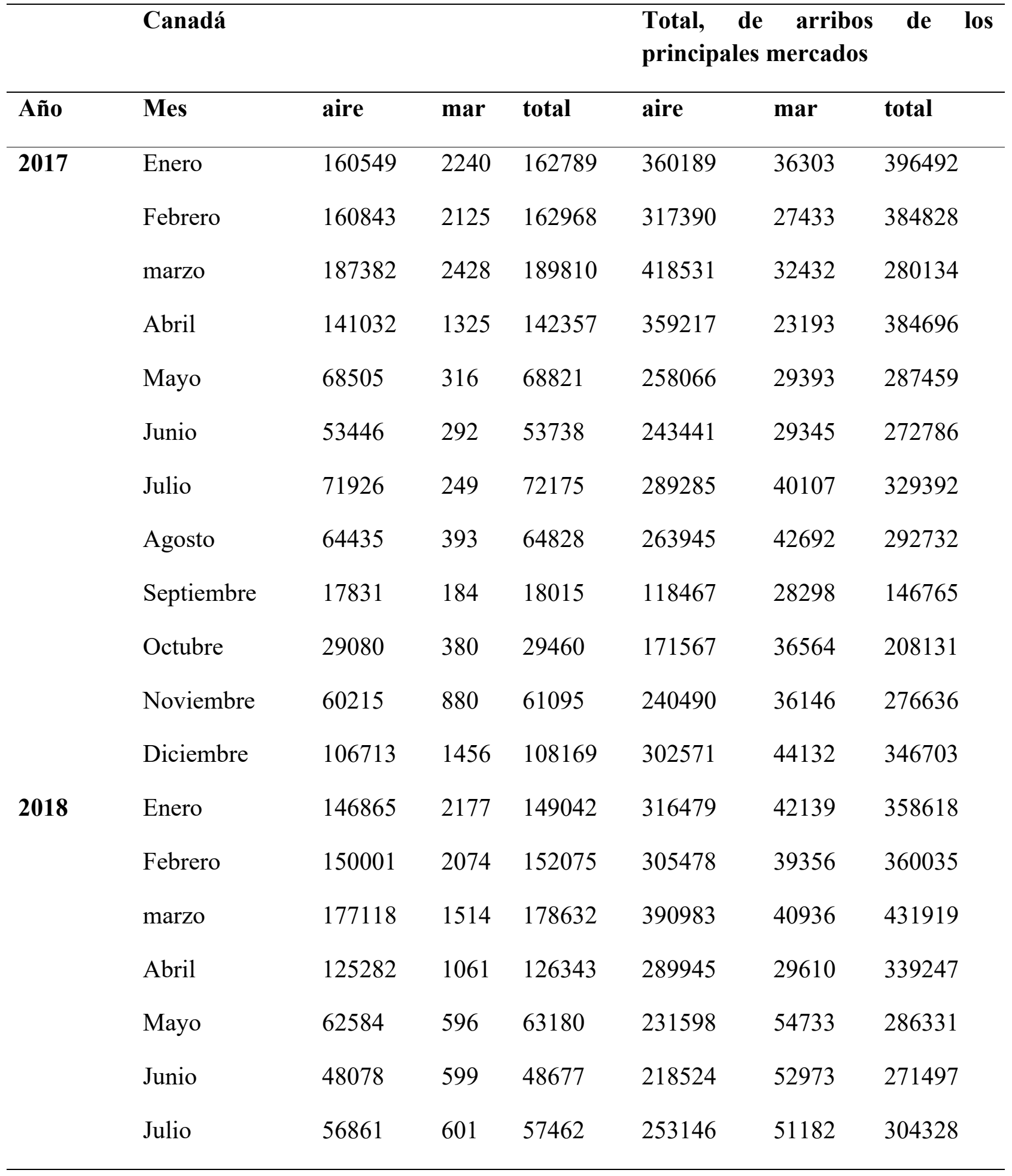


ISSN: 2773-7330 julio-septiembre, 2019

\begin{tabular}{|c|c|c|c|c|c|c|c|}
\hline & Agosto & 52522 & 825 & 53347 & 234358 & 62525 & 296883 \\
\hline & Septiembre & 31091 & 596 & 31687 & 167702 & 49395 & 217097 \\
\hline & Octubre & 41828 & 1256 & 43084 & 183285 & 55676 & 235370 \\
\hline & Noviembre & 84673 & 1378 & 86051 & 237028 & 50113 & 306869 \\
\hline & Diciembre & 118388 & 1662 & 120050 & 311919 & 51335 & 363254 \\
\hline 2019 & Enero & 156846 & 2201 & 159047 & 328701 & 63338 & 353420 \\
\hline & Febrero & 155094 & 1395 & 156489 & 322608 & 47676 & 373284 \\
\hline & marzo & 179122 & 1611 & 180733 & 365110 & 52572 & 439795 \\
\hline & Abril & 126815 & 1470 & 128285 & 314244 & 58858 & 373102 \\
\hline & Mayo & 57110 & 883 & 57993 & 224687 & 57944 & 282631 \\
\hline & Junio & 43354 & 94 & 43448 & 215032 & 8612 & 223644 \\
\hline & Julio & 53186 & 1 & 53187 & 240496 & 297 & 240793 \\
\hline & Agosto & 48935 & 2 & 48937 & 224258 & 272 & 224530 \\
\hline & Septiembre & 30817 & 3 & 30820 & 161184 & 162 & 161346 \\
\hline & Octubre & 40456 & 3 & 40459 & 181046 & 527 & 167146 \\
\hline & Noviembre & 91476 & 4 & 91480 & 273730 & 680 & 179874 \\
\hline & Diciembre & 129193 & 6 & 129199 & 317252 & 613 & 317865 \\
\hline
\end{tabular}

Fuente: Elaboración propia.

De estos datos se tomó como variable independiente el total de turistas canadienses, y como variable dependiente el total de turistas de los principales mercados llegados en cada mes:

V1: Total de turistas canadienses. V2: Total de turistas de los principales mercados.

Con la aplicación de la regresión múltiple el objetivo es determinar la dependencia de estas variables y la ecuación que las define entre ellas.

El diagrama de dispersión es el primer método aplicado para demostrar la dependencia entre estas dos variables: 
Tabla 2. Diagrama de dispersión resultados del SPSS.

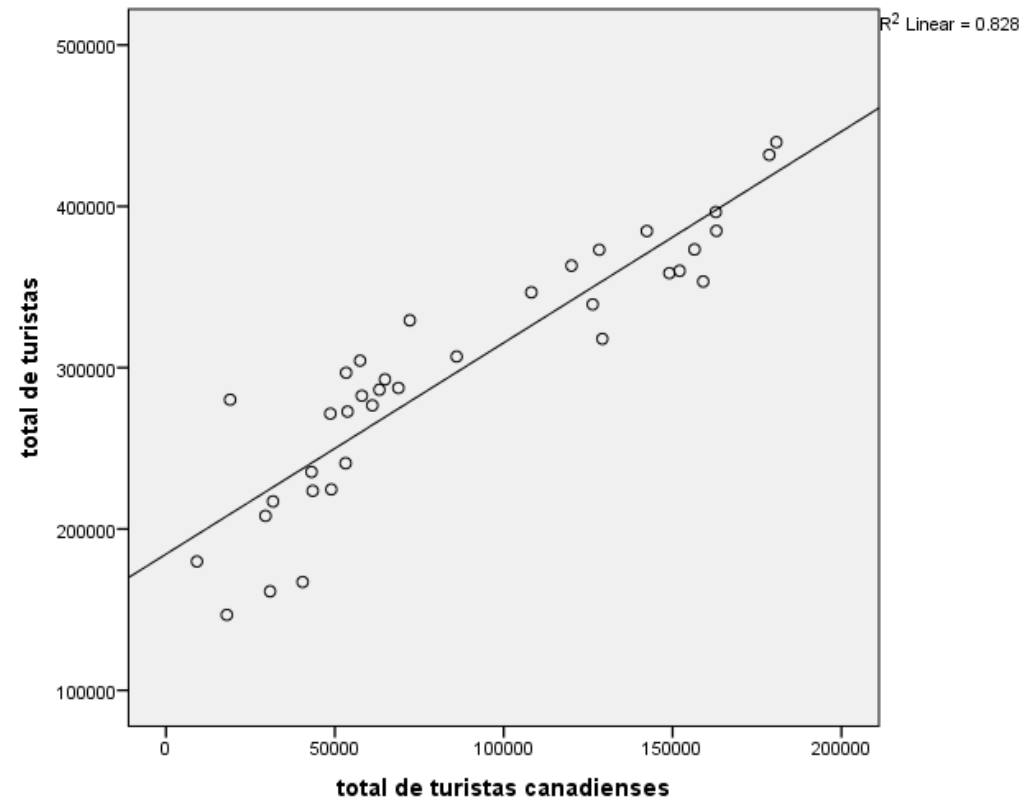

Fuente: Elaboración propia.

Este grafico demuestra la existencia de una correlación del tipo lineal positiva entre las variables estudiadas.

De igual manera el cálculo del coeficiente de correlación a partir del método de Pearson es de $\boldsymbol{\rho}=\mathbf{0 . 9 1}$, por lo que se puede afirmar que es una correlación muy alta, por estar en un intervalo entre 0.8 y 1 .

Una vez conocida la existencia de esta dependencia entre la llegada de turistas de los principales mercados y la llegada de turistas canadienses, prosigue determinar que tanto está determinada la variable dependiente por la variable dependiente. Para esto es necesario encontrar la ecuación lineal que las define.

El proceso de estimar la ecuación de regresión, describe el nombre de Ajuste de Curva, y consiste en estimar los valores particulares de los coeficientes de la ecuación seleccionada, a partir de los valores disponibles, es decir los estimadores de $\beta 0, \beta 1 \mathrm{y} \beta 2$, y este proceso se hará a partir del Método de los Mínimos Cuadrados Ordinarios(MMCO), que hace mínima la diferencia entre cada V2i(valores reales de V2) y el valor de V2(valores estimados), es decir, minimiza los errores de estimación. (Osorio Cuellar, 2016)

La forma de la función que relaciona a V1 y V2 puede ser de cualquier tipo. La aplicada en la investigación es la regresión simple, que busca relaciones en forma de líneas rectas. Siendo los parámetros $\beta 0$ y $\beta 1$ los parámetros a buscar.

$$
V 2=\beta 0+\beta 1 * V 1+E
$$


ISSN: 2773-7330

Resultados del modelo:

Tabla 3. Resultados

\begin{tabular}{ccccc}
\hline Model & R & $\begin{array}{c}\text { R } \\
\text { Square }\end{array}$ & $\begin{array}{c}\text { Adjusted } \\
\text { R Square }\end{array}$ & $\begin{array}{c}\text { Std. Error of } \\
\text { the Estimate }\end{array}$ \\
\hline 1 & $.910^{\text {a }}$ & .828 & .823 & 32013.805 \\
& a. constante: total de turistas canadienses \\
\hline
\end{tabular}

- $\mathrm{R}$ o $\rho=0.91$ correlación muy alta

- $\quad$ Error de estimación $(\mathrm{E})=32013.805$

Fuente: Elaboración propia.

Tabla 4. ANOVA ${ }^{\mathrm{a}}$

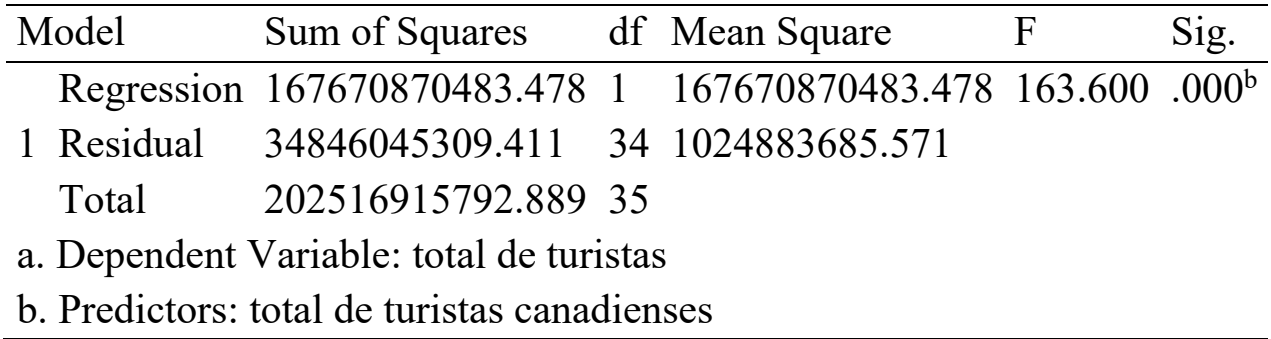

- $\mathrm{Sig}=0.00 \leq 0.05$

Fuente: Elaboración propia.

Tabla 5. Coefficientsa

\begin{tabular}{|c|c|c|c|c|c|c|c|c|}
\hline & \multirow{2}{*}{ Model } & \multicolumn{2}{|c|}{$\begin{array}{l}\text { Unstandardized } \\
\text { Coefficients }\end{array}$} & \multirow{2}{*}{$\begin{array}{c}\text { Standardized } \\
\text { Coefficients } \\
\text { Beta }\end{array}$} & \multirow{2}{*}{$\mathbf{t}$} & \multirow{2}{*}{ Sig. } & \multicolumn{2}{|c|}{$\begin{array}{l}\text { 95.0\% Confidence } \\
\text { Interval for B }\end{array}$} \\
\hline & & B & $\begin{array}{l}\text { Std. } \\
\text { Error }\end{array}$ & & & & $\begin{array}{l}\text { Lower } \\
\text { Bound }\end{array}$ & $\begin{array}{l}\text { Upper } \\
\text { Bound }\end{array}$ \\
\hline \multirow[t]{2}{*}{1} & (Constant) & 184447.872 & 10334.774 & & 17.847 & .000 & 163445.084 & 205450.659 \\
\hline & $\begin{array}{l}\text { total de } \\
\text { turistas } \\
\text { canadienses }\end{array}$ & 1.310 & .102 & .910 & 12.791 & .000 & 1.102 & 1.518 \\
\hline & pendent Vari & le: total de tu & & & & & & \\
\hline
\end{tabular}

- $\boldsymbol{\beta 0}=184447.872$

- $\boldsymbol{\beta} 1=1.31$

Fuente: Elaboración propia.

Intervalos de confianza al $0.95 \%$ para $\beta$ :

- $163445.084 \leq \boldsymbol{\beta 0} \leq 205450.659$

- $1.102 \leq \boldsymbol{\beta} 1 \leq 1.518$ 
La aplicación del modelo arroja como resultados las ecuaciones:

- Ecuación 1: Estimación puntual: V2=184447.872+1.31*V1

- Ecuación 2: Intervalos de estimación: V2=184447.872+1.31*V1+/- 32013.805

Basado en que los resultados que arroja el empleo del modelo dan un estimado probable es recomendable utilizar la ecuación 2 a la hora de hacer predicciones ya que a pesar de que la correlación entre las variables es muy alta, no es un ajuste perfecto, por lo que su estimación puntual está sujeta a errores.

\section{Ejemplo:}

Se selecciona de la tablal el valor de V1 correspondiente al mes de diciembre del año 2018 y se sustituye en ambas ecuaciones para estimar la llegada de turistas internacionales (V2) y compararla con el valor real (V2i)

$$
\mathrm{V} 1=120050 \mathrm{~V} 2 \mathrm{i}=363254
$$

- Ecuación 1:

$$
\mathrm{V} 2=184447.872+1.31 * \mathbf{1 2 0 0 5 0}
$$

$R / \mathrm{V} 2=\mathbf{3 4 1 7 1 3 . 3 7 2}$

- Ecuación 2:

$$
\begin{gathered}
\boldsymbol{V} \mathbf{2}=184447.872+1.31 * \mathbf{1 2 0 0 5 0}+/-32013.805 \\
R / \mathbf{3 0 9 6 9 9} \leq \boldsymbol{V} \mathbf{2} \leq \mathbf{3 7 3 7 2 7}
\end{gathered}
$$

$\boldsymbol{R} /$ Para una llegada de 120050 turistas canadienses en el mes de diciembre de 2018 se estima un total de turistas internacionales que oscile entre los 300000 y los 3700000 . (Valor real 363254 turistas internacionales).

Como se observa en ejemplo la estimación se corresponde con el valor real de la variable dependiente para el mes de diciembre de 2018.

De esta forma queda demostrada la posibilidad de estimar las llegadas internacionales mensuales a partir de la llegada de turistas canadienses a la isla con la utilización de la Ecuación 2. Por lo tanto, garantizando un comportamiento estable de este mercado se puede estabilizar el comportamiento global de turistas en el país.

A pesar de la veracidad de lo anteriormente descrito, las fluctuaciones de llegadas en el turismo no son predecibles, y los resultados propuestos pertenecen a un escenario medio a 
partir del estudio del comportamiento de las variables. Pero esto no quita la posibilidad de la llegada de situaciones casi ideales como la vivida en el año 2016, en donde el crecimiento turístico fue del $24 \%$, rompiendo las predicciones de crecimiento de solo un $6 \%$ estimada para ese año. Así como la gestación de escenarios totalmente desfavorables, como las afectaciones producto del bloqueo a las que la nación cubana está siendo sometida en la actualidad.

Para la predicción de estos dos tipos de escenarios el modelo ofrece intervalos de un $0.95 \%$ de confianza para los estimadores $\beta 0 \mathrm{y} \beta 1$, con los que se puede determinar la probabilidad de llegadas internacionales.

\section{Intervalos de confianza al $0.95 \%$ para $\beta$ :}

- $163445.084 \leq \boldsymbol{\beta 0} \leq 205450.659$

- $1.102 \leq \boldsymbol{\beta} 1 \leq 1.518$

Con estos la ecuación dos queda descritas de las siguientes dos formas:

Ecuación 3: Escenario pesimista:

- $\mathrm{V} 2=163445.084+1.102 * V 1+/-32013.805$

Ecuación 4: Escenario optimista:

- $V 2=205450.659+1.518 * V 1+/-32013.805$

De esta manera se obtienen 3 ecuaciones (ecuación 2, ecuación 3, ecuación 4) que permiten la estimación mensual de la cantidad de turistas llegados de los principales mercados a Cuba a partir de la llegada de turistas canadienses, valorando los posibles escenarios a los que puede estar sometida la nación caribeña en los próximos años.

\section{Conclusiones.}

A partir de los objetivos propuestos la presente investigación puede llegar a las siguientes conclusiones:

- La aplicación de herramientas estadísticas e informáticas permitió el correcto análisis de los datos suministrados para la investigación.

- Existe una relación de dependencia entre la llegada de turistas canadienses y la llegada total de turistas proveniente de los principales mercados que puede ser medida matemáticamente a partir de las ecuaciones propuestas. 


\section{Referencias bibliográficas.}

Fernández, G. (2015). Un Modelo de programacion lineal para la optimizacion de la ganancia en un estaurant y su comparacion con otras tecnicas utilizadas de perfeccionamiento del menu. La Habana: Univercidad de la Habana.

Guevara, A., Aguayo, M., Aguayo, A., \& Araque, F. (2013). Informática aplicada al turismo. Ediciones Pirámide, 312.

Ivars Baidal, J., Solsona Monzonís, J., \& Giner Sánchez, D. (2016). Gestión turística y tecnologías de la información. Documents d'Anàlisi Geogràfica 2016, 327-346.

Martín Fernández, R. (2006). Principios, organización y práctica del turismo. La Habana.

Méndez Álvares, C. E. (2001). Metodología. Diseño y desarrollo del proceso de investigación., 137.

Millán Gasca, A. (2006). La aplicación de las Matematicas a los problemas de administración y control: Antecedentes Históricos. ILUIL, vol.26, 929-961.

Organización Mundial del Turismo (OMT). (26 de mayo de 2017). Apuntes de Metodología de la Investigación en el Turismo. Obtenido de e-unwto.org: http:/www.eunwto.org/doi/book/10.18111/9789284404889 - Friday, May 26, 2017 7:39:52 PM Secretaría de Turismo IP Address:189.204.93.100

Osorio Cuellar, P. B. (2016). Programación lineal para la distribución de viajes en. Lima: Universidad Nacional de San Marcos, Facultad de Ciencias Matemáticas.

Vázquez Alfonso, Y. (2018). Banco de datos turísticos para el monitoreo y toma de decisiones en entidades del Turismo. La Habana: Facultad de Turismo.

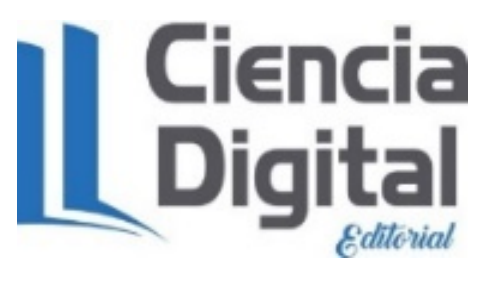




\section{Para citar el artículo indexado}

Zulueta, D. A., \& Lemes Reyes, M. (2020). Influencia del mercado canadiense en el turismo cubano. AlfaPublicaciones, 1(2), 30-40. https://doi.org/10.33262/ap.v1i2.8

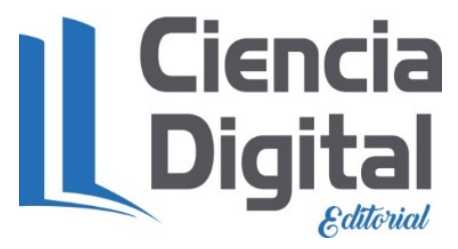

El artículo que se publica es de exclusiva responsabilidad de los autores y no necesariamente reflejan el pensamiento de la Revista Alpha Publicaciones.

El artículo queda en propiedad de la revista y, por tanto, su publicación parcial y/o total en otro medio tiene que ser autorizado por el director de la Revista Alpha Publicaciones.
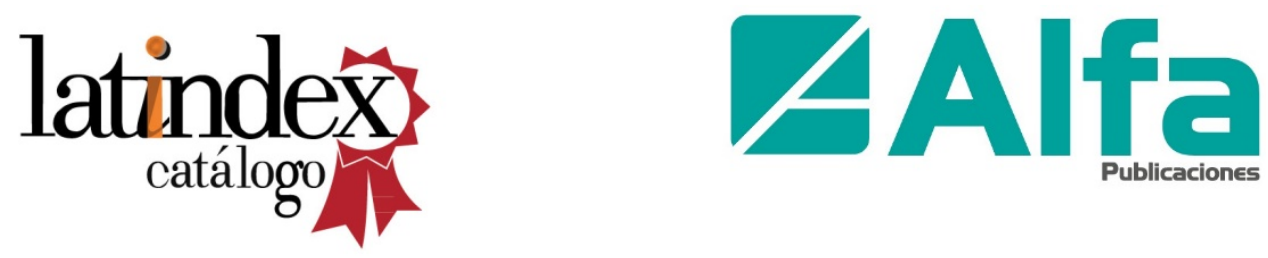